\title{
El valor percibido por el cliente y el comportamiento del consumidor como constructos paralelos a las Leyes de Gossen ${ }^{\dagger}$
}

\author{
The value perceived by the customer and the consumer \\ behavior as parallel constructs to Gossen's laws \\ $\mathrm{O}$ valor percebido pelo cliente e o comportamento do \\ consumidor como construçóes paralelas as Leis de Gossen
}

Dr. Divvio Gallegos Paniagua*

\section{RESUMEN}

La finalidad del artículo es establecer la relación entre los principios económicos de la conducta del consumidor con las bases mercadológicas del comportamiento del consumidor y el valor percibido por el cliente, para lo cual se exponen las Leyes de Gossen como fundamento. Se realiza revisando inicialmente los principios previamente mencionados, para luego abordar los conceptos de intensidad de las necesidades, valoración de los productos, precios y decisiones de compra. Finalmente, se exponen las Leyes correspondientes y se presentan hechos publicados mediáticamente como ejemplos de la vigencia de la propuesta de Gossen y de la relación entre los principios de la conducta y el comportamiento del consumidor.

Palabras clave: intensidad de las necesidades, satisfacción, valor, valor percibido por el cliente.

\begin{abstract}
The purpose of this paper is to establish the relationship between economic principles and market principles of consumer behavior and costumer perceived value, the Laws of Gossen exposed as the medium: first a review of the above principles and then an approach the current concepts of needs assessment of the products, prices for purchasing decisions. Finally, the corresponding laws are presented, and some published facts are presented as examples of the effect Gossen proposed and the relationship between the conduct and behavior of the consumer is presented.
\end{abstract}

Keywords: intensity needs, satisfaction, value, costumer perceived value.

\section{RESUMO}

A finalidade do artigo é o de estabelecer a relação entre os princípios económicos da conduta do consumidor com as bases mercadológicas do comportamento do consumidor e o valor percebido pelo cliente, para o qual se expóem as Leis do Gossen como o fundamento. Realiza-se uma revista inicial nos princípios previamente mencionados para logo abordar os conceitos de intensidade das necessidades, valoraçẫo dos produtos, preços, decisóes de compra. Finalmente se expóem as Leis

Código J.E.L.: M 390, M 210, M 120.

* Mexicano, Doctor en Administración, Licenciado en Economía, Profesor e Investigador, Instituto Tecnológico de Morelia, Morelia, México. Correspondencia con el autor: divviotec@gmail.com. 
El valor percibido por el cliente y el comportamiento del consumidor como constructos paralelos a las Leyes de Gossen / D. Gallegos

correspondentes e se apresentam fatos publicados midiaticamente como exemplos da vigência da proposta de Gossen e da relação entre a conduta e o comportamento do consumidor.

Palavras-chave: intensidade das necessidades, satisfação, valor, valor percebido pelo cliente.

\section{Introducción}

La Economía y el Marketing se relacionan en el presente artículo en tres de sus bases conceptuales: la Conducta del Consumidor, el Comportamiento del Consumidor y el Valor Percibido por el Cliente, tomando como base teórica y elemento de relación un análisis propuesto en el siglo XIX por el destacado marginalista Hermann Heinrich Gossen, denominado Leyes de Gossen en reconocimiento al autor.

El abordaje del tema se hace, inicialmente, revisando literatura acerca de las teorías de la conducta del consumidor y la del comportamiento del consumidor, para a continuación realizar indagatoria de puntos comunes en conceptos tales como necesidades, motivación al consumo, valoración y precio, para posteriormente establecer una idea común de toma de decisión de compra por el público, paralelo en lógica al denominado Valor Percibido por el Cliente (VPC).

Se finaliza con ejemplos, a manera de comprobación de la validez del supuesto, es decir, se revisa información mediática para establecer la vigencia de las Leyes de Gossen como base de explicación de diversos principios mercadológicos del comportamiento del consumidor.

En el primer tema se expone la metodología utilizada, resaltando la modalidad de ejemplificación mediáticamente cuando los fenómenos observados son públicos.

El tema dos presenta la conceptualización que habrá de servir como herramienta de análisis en el desarrollo subsecuente del artículo, para abordar en el tercer tema dos de las tres Leyes de Gossen, mismas que son el antecedente de la teoría de la conducta del consumidor, uno de los pilares del enfoque subjetivo del valor, base de la teoría económica moderna.

Finalmente, en el cuarto tema se presentan evidencias de productos y empresas en las que se observan diversos aspectos que muestran la vigencia de los principios de Gossen en los fenómenos cotidianos del mercado.

\section{Método}

El abordaje del tema parte de la revisión teórica de autores de las ciencias económicas como de estrategias mercadológicas, hasta presentar evidencias mediáticas como ejemplos de hechos actuales y cotidianos. 
Por el enfoque utilizado, la investigación presentada es hipotética deductiva, utilizando tres conceptos básicos como lo son conducta del consumidor, valor percibido por el cliente y comportamiento del consumidor, correspondientes a ciencias de enfoques diferentes (el primero de la Economía y los dos últimos de Mercadotecnia). El logro consiste en relacionar ambos conceptos para explicar las decisiones de compra de valores creados para el cliente.

El corte teórico del estudio se complementa con ejemplos de hechos sucedidos recientemente (primera y segunda década del siglo XXI) y publicados en los medios, como recursos que permiten deducir la validez y vigencia de las ideas del autor referente: Hermann Henrich Gossen (1810-1858).

El proceso de análisis se realiza acudiendo a diversos autores que abordan un mismo tema o idea; de ellos se toman los aspectos más útiles para el entendimiento del tema estudiado. Finalmente, se culmina el proceso al llegar a la síntesis por medio de un posicionamiento en cada apartado, construido en base a los diversos autores consultados.

\section{El comportamiento del consumidor}

Los estudios de mercado suelen plantearse como retos para la introducción de nuevos productos en los mercados o bien la para consolidación en los mismos, por lo que es fundamental conocer la aceptación de los satisfactores por el público.

A continuación se presentan los conceptos e ideas básicas sobre el comportamiento del consumidor y la conducta del consumidor, que serán las "herramientas" para el desarrollo del artículo, destacando finalmente la importante relación entre conceptos de las ciencias económicas y de las estrategias mercadológicas.

El comportamiento del ser humano hacia la satisfacción de sus necesidades ha sido motivo de diversos estudios, ya que la posibilidad de lograr inducir a determinada población o grupo de ella a tomar decisiones de compra puede ser un gran avance para las empresas. Es en especial en Economía y en Marketing en donde dicho tema se aborda por ser un aspecto fundamental de su objeto de estudio. Así “... la ańeja discusión de si la mercadotecnia crea o no las necesidades, a través de la manipulación psicológica, tiene importantes implicaciones para el estudio de la conducta de los consumidores" (Sahui, 2008:3).

Por conducta del consumidor se debe entender todo un conjunto de actos económicos, de acciones de las personas encaminadas a satisfacer sus necesidades y su toma de decisiones sobre adquisición, uso y consumo de productos, tanto tangibles como 
El valor percibido por el cliente y el comportamiento del consumidor como constructos paralelos a las Leyes de Gossen / D. Gallegos

intangibles. "Al conjunto de acciones que realiza se le conoce como conducta del consumidor" (Méndez, 2007:104).

Un fundamento para realizar los diversos estudios sobre el tema es reconocer lo que da lugar a los esfuerzos que realizan los seres humanos para consumir, lo que es reconocible a primera vista y atribuible a sus necesidades, es decir, aquello que mueve al ser humano, puesto que "para entender el comportamiento de los consumidores primero hemos de preguntarnos por qué un individuo realiza una acción. La respuesta es 'porque siente una necesidad"' (Stanton, Etzel y Walker, 2000:131).

\section{a) Conceptos}

Contar con un concepto de comportamiento de consumidor es determinante. En general hay muchas coincidencias sobre este aspecto. Se converge en la búsqueda de la satisfacción de necesidades como motivo: "es el comportamiento que los consumidores muestran al buscar, comprar, utilizar, evaluar y desechar los productos y servicios que, consideran, satisfarán sus necesidades" (Schifman y Kanuk, 2010:8).

Acerca de las necesidades, éstas se solventan mediante satisfactores, siendo entonces que el comportamiento del consumidor se refiere a actos, acciones, relaciones de los individuos en la búsqueda de obtener y usar bienes y servicios, o sea productos, que permitan satisfacer sus necesidades (Fisher, 1997). Es entonces que producir, así como consumir satisfactores, son actividades fundamentales en la vida cotidiana de las sociedades.

El campo del comportamiento del consumidor es sumamente amplio y se comprende como "... el estudio de las formas en que el consumidor toma sus decisiones de compra de productos que le permitan satisfacer sus necesidades y deseos” (Atoche, 2010:L.5), abarcando también aspectos más específicos como aquello que se "... refiere al análisis de: hábitos de compra/consumo, frecuencia de uso/consumo” (Atoche, 2010:L.5). Abarcando también, por consecuencia, aquellas decisiones referentes a generar los productos tangibles e intangibles capaces de satisfacer las necesidades.

Comportamiento del consumidor y conducta del consumidor son dos conceptos estrechamente relacionados entre sí; el primero como construcción conceptual del Marketing y el segundo de la Economía; el primero, más reciente que el segundo, el cual logra una descripción del porqué de las decisiones de comprar o no del individuo (Arce, Gallegos y Avendaño, 2015).

La propuesta de VPC es realizada por Valeri Zeithaml hacia 1988 y, a partir de esa idea, "...se han sucedido un gran número de aportaciones que tratan de acotar y dar contenido al término que nos ocupa” (Martín, Barroso y Martín, s. f.:47). 


\section{b) Necesidades y deseos}

Las necesidades son el punto de partida que desencadena el conjunto de acciones para satisfacerlas, por lo que se puede afirmar que "toda conducta comienza con una necesidad" (Stanton et al., 2000:131), y aunque, sin lugar a dudas, la necesidad obedece a su vez a una serie de factores "...casi siempre la decisión de compra es resultado de un conjunto de factores no solamente psicológicos sino también culturales, económicos y sociales" (Sahui, 2008:3), todo eso conforma a las necesidades, por lo cual este concepto es fundamental.

Además de lo anterior, la necesidad suele ser lo que motiva la conducta del consumidor, la que desencadena un conjunto de actos y acciones orientadas al disfrute de satisfactores, siendo entonces que la motivación "es la búsqueda de la satisfacción de la necesidad, la cual, generalmente, se centra en la realización de actividades específicas tendientes a disminuir la tensión producida por la necesidad" (Siliceo, s. f.:91).

Así, una necesidad intensa se transforma en deseo, el que, a su vez, da como respuesta una conducta de esfuerzo por lograr disponer de un satisfactor. "Para la psicología, las motivaciones son el conjunto de factores que impulsan el comportamiento de los seres humanos hacia la consecución de un objeto. Por ejemplo, una persona tiene muchas necesidades en cualquier momento" (Sahui, 2008:8).

Las necesidades bajo la condición de deseo se pueden entender como una situación extrema y en consecuencia altamente motivante, debido a que ciertas necesidades "... son el resultado de estados fisiológicos de tensión, como el hambre, la sed y la incomodidad. Otras, resultado de los estados psicológicos de tensión, como la necesidad de reconocimiento, estimación o pertenencia" (Sahui, 2008:8).

Es, entonces, la satisfacción de sus necesidades lo que motiva al ser humano a decidir la compra de un satisfactor: todo ser humano requiere satisfacer sus necesidades de alimento, vestido, vivienda y demás para sobrevivir; de dicha satisfacción depende su existencia material y por ello la gran mayoría de sus acciones estarán encaminadas a tal fin (Arce et al., 2015).

\section{c) Elecciones del consumidor}

Desde luego que los actos y acciones económicas del ser humano van más allá de simples respuestas a sus instintos, siendo en cambio procesos más amplios que los de estímulo-respuesta, que son influenciados por la condición individual y el contexto social de la persona. "El comportamiento de compra de los consumidores finales puede examinarse usando un modelo de cinco partes: el proceso de decisión de la compra, 
El valor percibido por el cliente y el comportamiento del consumidor como constructos paralelos a las Leyes de Gossen / D. Gallegos

la información, los factores sociales y grupales, los factores psicológicos y los factores situacionales" (Stanton et al., 2000:140).

Debido a lo anterior, tanto el impulso instintivo y la racionalidad operan en los procesos que llevan a la persona a actuar para disponer de los satisfactores. "Las etapas del proceso de decisión de compra son: reconocimiento de una necesidad, identificación de alternativas, evaluación de alternativas, decisiones de compra y relativas a la compra, y comportamiento post compra” (Stanton, Etzel y Walker, 2000:140).

\section{d) Conducta y comportamiento del consumidor}

Para que una necesidad se traduzca en motivo para la acción debe ser altamente significativa en el momento presente del ser humano. La toma de decisiones tendrá como base una carga de intensidad lo suficientemente importante como para llevar a la acción al consumidor. "La mayoría de estas necesidades no son lo bastante intensas para motivar a la persona a actuar en un momento dado, pero una necesidad se convierte en un motivo cuando alcanza un nivel adecuado de intensidad" (Sahui, 2008:8).

Aunque algunos autores limitan la decisión de demandar a ingresos, precio de bienes sustitutos, precio de bienes complementarios, el gasto de los consumidores y expectativas de precios (Ramos, 2004), la necesidad del satisfactor que corresponda así como la intensidad de dicha necesidad es fundamental, la cual se entenderá como “...un proceso en el cual interviene el deseo del individuo de cubrir la brecha entre lo que se tiene actualmente y lo que se quisiera tener" (Siliceo, s. f.:90).

El concepto de conducta del consumidor -tema explorado por la ciencia económica-es un factor básico para entender el comportamiento del consumidor -concepto básico de las ciencias mercadológicas-, que explica las decisiones de compra de los agentes de mercado y que permite a las empresas desarrollar las estrategias de introducción de bienes y servicios en los mercados, así como consolidarlos en los mismos.

\section{Las Leyes de Gossen}

En el tema antecedente se presentaron los diversos conceptos que se utilizan en este artículo, a manera de contar con las ideas básicas que permitan una comunión semántica. Conducta y comportamiento del consumidor, escasez, valor y precio han sido definidos. Cabe a continuación presentar la manera como Gossen explicó su formación de esas variables, así como su relación, hasta culminar con una explicación de la toma de decisión de compra por el consumidor. 


\section{a) Las necesidades y la escasez}

Las necesidades son estudiadas por la psicología desde la perspectiva motivacional, entendiéndose como "...un estado interno que es menos que satisfactorio..." "...por la falta de algo..." "para el bienestar" (Carver y Sheier, 2014:84), un algo de lo cual se carece, es decir, que es escaso en un determinado momento y condición personal.

La necesidad puede llegar a ser el motivo de las acciones del individuo, siempre que esta necesidad sea estimulada internamente o externamente por lo que "...un motivo es una necesidad lo suficientemente estimulada como para impulsar al individuo a buscar la satisfacción" (Stanton, Etzel y Walker, 2000:131).

Motivación y necesidad están igualmente regidas a la vez que relacionadas por su respectiva intensidad. El grado de motivación en búsqueda de satisfacción guarda relación directa con la intensidad de la necesidad, de manera que "una necesidad se convierte en un motivo cuando llega a un determinado grado de intensidad" (Kotler y Armtrong, 1996:172). Un grado extremo de necesidad existe por la escasez de algo para una persona.

Figura 1. De la necesidad al comportamiento.

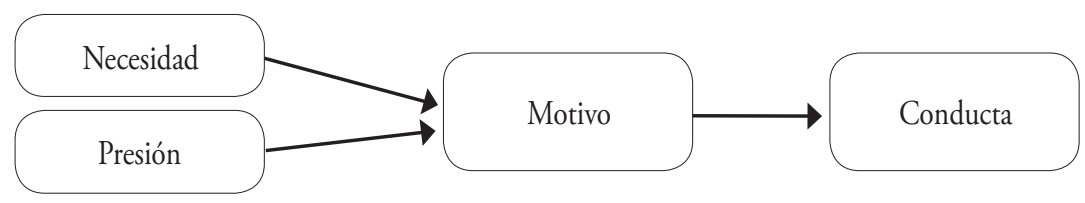

Los estados de necesidad interna y la presión externa influyen en la motivación para realizar acciones específicas, lo que a su vez se reflejará en un comportamiento evidente.

Fuente: elaboración propia en base a Craver y Sheier (2014:86).

En principio, entonces, las necesidades anteceden a los motivos y los generan, por lo que entre la necesidad y la acción para satisfacerla media la motivación, siendo una manera de comprender que "las necesidades son como motivación, o sea como fuerza impulsora dentro de los individuos que los empuja a la acción. Esta fuerza impulsora se genera por un estado de tensión que existe como resultado de una necesidad insatisfecha” (Schifman y Kanuk, 2010:87).

Así entonces, la necesidad bien pudiera entenderse como un estado de desequilibrio biopsicosocial por la carencia o escasez de algo, que motiva al ser humano a actuar a fin de obtener aquello que elimine dicho desequilibrio -o sea satisfacer una necesidadmediante ese algo que bien puede denominarse satisfactor. Ese estado de desequilibrio causa una tensión interna y con el exterior, que desencadena las acciones en búsqueda de 
El valor percibido por el cliente y el comportamiento del consumidor como constructos paralelos a las Leyes de Gossen / D. Gallegos

obtener los satisfactores necesarios que permitan recuperar el equilibrio biopsicosocial, también entendido como bienestar.

\section{b) Concepto de utilidad}

La utilidad está estrechamente relacionada con las necesidades y la toma de decisiones de consumo de productos. La utilidad de los productos está determinada por la intensidad de las necesidades, en tanto que la utilidad determina a su vez el valor de los productos, considerando que la utilidad "es la capacidad que tienen los bienes para satisfacer necesidades humanas” (Méndez, 2007:11). Conforme mayor capacidad para satisfacer determinada necesidad, mayor utilidad, y conforme mayor utilidad, mayor valor, esto es, "utilidad, beneficio o satisfacción que obtiene una persona a partir del consumo de bienes o servicios" (Parkin, 2014).

Siendo que la utilidad marginal es el "cambio en la utilidad total, resultante del incremento de una unidad en la cantidad consumida de un bien” (Parkin, 2014), este aspecto de la utilidad determina el límite del consumo de un bien, es decir, la cantidad consumida está determinada por aquella utilidad marginal, la cual a su vez está determinada por la intensidad de la necesidad, siendo que conforme aumenta el consumo, se reduce la intensidad de la necesidad y, por consiguiente, también se reduce la utilidad marginal, lo que le da su característica decreciente, "...tendencia de la utilidad marginal a disminuir a medida que se incrementa la cantidad consumida de un bien” (Parkin, 2014).

\section{c) El valor}

Entre la intensidad de las necesidades y el valor asignado a cada unidad de un satisfactor, existe una relación estrecha, en tanto éste es el “... grado de preferencia que una persona tiene por un producto o servicio” (Robles y Alcérreca, 2000:39), la utilidad se erige como variable interviniente puesto que la intensidad de la necesidad determina la utilidad y ésta, a su vez, determina el valor.

En tanto, el valor determina el constructo social de precio justo. "Se refleja en el máximo precio que una persona estaría dispuesta a pagar por el bien dada la percepción de sus características" (Robles y Alcérreca, 2000:39). Así el valor es, a su vez, una variable interviniente respecto a la intensidad de las necesidades.

En base a lo previamente anotado, otros autores como Kotler y Armstrong (1996) denominan el término como un instrumento de aplicación o a partir de su utilidad: "instrumento principal para identificar maneras de crear más valor para el cliente". Llevado al campo del emprendedurismo, "un concepto que recientemente se ha vuelto importante para los emprendedores es definir con claridad la forma como vas a generar una solución que 
le aporte valor agregado al cliente y la forma como vas a ganar dinero con tu negocio" (Hilarión, 2013:15). Una variable muy importante para determinar el precio.

Igualmente en el campo del marketing, la utilidad y su consecuente valor permiten tomar decisiones trascendentes, ya que "si el precio es mayor al de la competencia, debes asegurar que tu propuesta de valor y beneficios que el cliente percibe con el producto o servicio de tu empresa son superiores a los de la competencia" (Hilarión, 2013:15).

Con una visión más amplia, el valor creado para el cliente permite que "con una fuerza laboral diversa y una cultura organizacional positiva en marcha, muchos gerentes consideran que sus empresas podrán:

- Desarrollar productos y servicios para nuevos mercados.

- Atraer una gama de clientes más amplia.

- Mejorar la satisfacción del cliente y aumentar los negocios con la conservación de los clientes" (Helliegel, Jackson y Slocum, 2004:501).

Un constructo desarrollado por los mercadólogos como lo es el de valor percibido por el cliente, tiene sus antecedentes sin lugar a dudas en Gossen. Tal se define como "... la evaluación global por parte del mismo (el cliente), de la utilidad y desempeño de un producto, con relación a la competencia y basada en la percepciones de lo que se recibe y de lo que se da a cambio" (Baby y Uribe, 2014:28). Dicho concepto data, de acuerdo con los autores, de finales de los ańos setenta.

\section{d) Precio}

El precio, como coeficiente de intercambio de dinero por producto, es un instrumento útil para adquirir, vender y calcular el valor comercial, entre otros usos, si "por precio se entiende la cantidad por la que se ofrece o se intercambia un determinado producto o servicio" (Robles y Alcérreca, 2000:155).

Para el intercambio, disponer de precios que reflejen el valor asignado facilita el intercambio y, por consecuencia, el acceso a productos con lo que los satisfactores toman el carácter económico, esto es, un “... bien que tiene utilidad pero es escaso, en relación con la demanda, por lo que requiere de algún método de racionamiento, por ejemplo, cobrando un precio por él” (Robles y Alcérreca, 2000:80).

\section{e) Las dos Leyes de Gossen}

Hermann Henrich Gossen (1810-1858) es considerado como uno de los más destacados marginalistas, sin embargo, quizás por la sencillez de sus planteamientos lo hacen poco 
El valor percibido por el cliente y el comportamiento del consumidor como constructos paralelos a las Leyes de Gossen / D. Gallegos

conocido. "El aporte de Gossen se refiere a la teoría de la utilidad. Su obra, publicada en 1854, Entwicklung des Gesetze des Menschlichen Verkehrs, pasa completamente desapercibida hasta que Jevons la descubre en 1878 y hace de Gossen uno de los pioneros" (Gómez, s. f.: s. p.). Por lo anterior, desde el espacio filosófico es considerado como utilitarista.

Hoy día las ideas de Gossen son expresadas en diversos argumentos -quizá aún sin saberlo- por importantes autores de la mercadotecnia, lo que da una idea de su trascendencia. Un ejemplo de ellos es la cita siguiente: "el hombre tiene muchas necesidades latentes que no originan comportamiento por no ser lo bastante intensas" (Stanton, Etzel y Walker, 2000:131).

\section{i. Primera Ley de Gossen}

La llamada primera Ley de Gossen relaciona a la intensidad de la necesidad con la satisfacción de manera tal que conforme más se consume de un producto, menor será la intensidad de la necesidad, ya que "toda necesidad disminuye en intensidad a medida que se satisface. Pero ninguna unidad de una cantidad dada de riqueza, por ser intercambiable con las demás unidades, puede tener un valor superior al de la que se aplica a la satisfacción de la más débil de las necesidades satisfechas" (James, 1966:187).

Figura 2. La intensidad de la necesidad en relación inversa a la cantidad consumida.

$$
\mathrm{Y}=\mathrm{f}(\mathrm{X})
$$

Intensidad de la Necesidad $=f($ Cantidad $)$

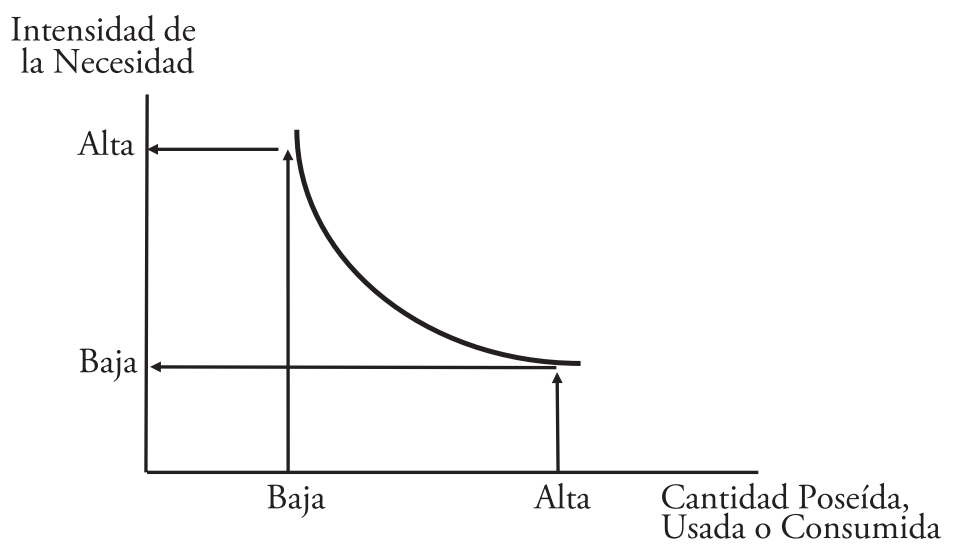

Fuente: elaboración propia.

La gráfica anterior establece la relación descrita en la primera Ley de Gossen, por lo que conforme es menor la cantidad consumida o poseída de un satisfactor, la intensidad de la necesidad es mayor. Como se observa en la gráfica siguiente, esto tiene consecuencias en la utilidad. 
Figura 3. Relación entre la intensidad de la necesidad y la utilidad.

$$
\mathrm{Y}=\mathrm{f}(\mathrm{X})
$$

Utilidad $=\mathrm{f}($ Intensidad de la Necesidad $)$

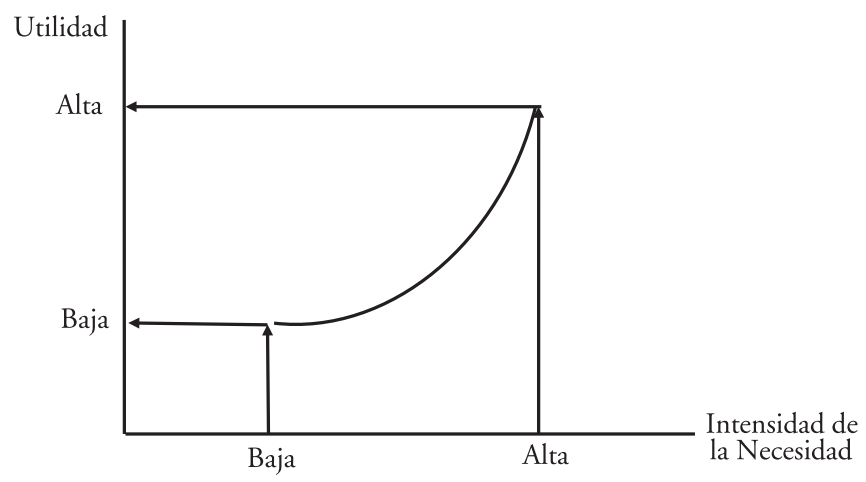

Fuente: elaboración propia.

La figura 3 describe gráficamente que conforme mayor es la intensidad de la necesidad, mayor es la utilidad del satisfactor, lo que a su vez relaciona la utilidad con la cantidad consumida, como a continuación se ilustra.

Figura 4. Relación entre la utilidad y la cantidad consumida.

$$
\begin{aligned}
\mathrm{Y} & =\mathrm{f}(\mathrm{X}) \\
\text { Utilidad } & =\mathrm{f}(\text { Cantidad })
\end{aligned}
$$

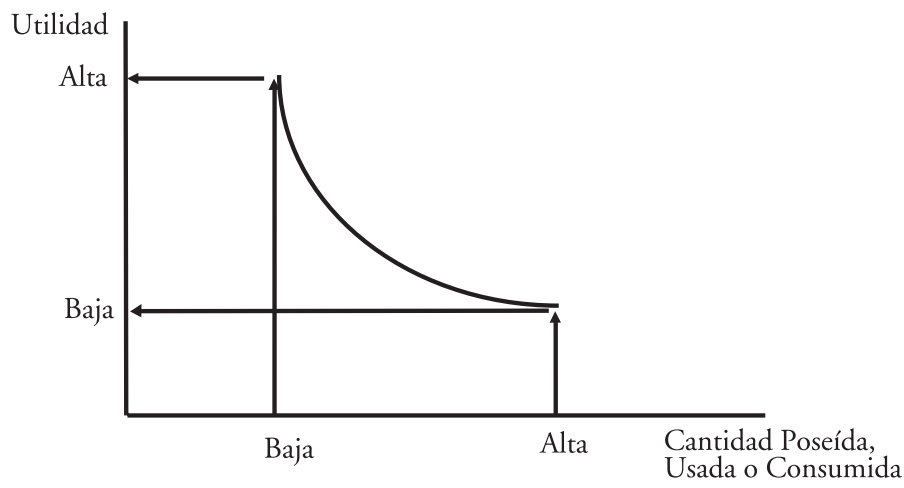

Fuente: elaboración propia.

En la figura 4 se explica gráficamente que conforme mayor es la cantidad consumida, baja la intensidad de la necesidad y, por consecuencia, baja la utilidad que cada unidad adicional de un satisfactor pueda tener, esto es, baja la utilidad marginal cada nueva unidad del producto de que se trate.

Lo descrito previamente se reflejará necesariamente en la valoración que hace el consumidor acerca del producto, lo cual se expone a continuación. 
El valor percibido por el cliente y el comportamiento del consumidor como constructos paralelos a las Leyes de Gossen / D. Gallegos

Figura 5. Relación entre la valoración y la cantidad consumida.

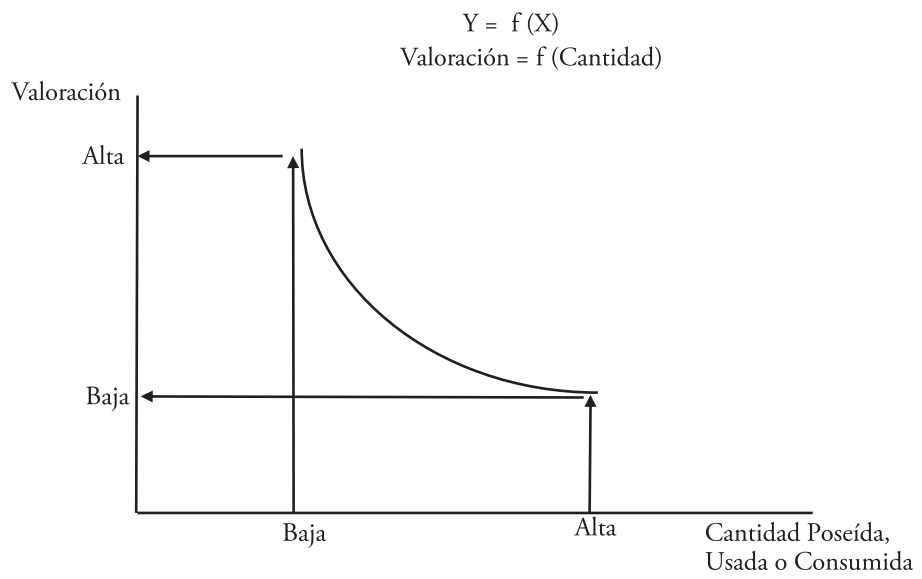

Fuente: elaboración propia.

La gráfica anterior describe que el valor reconocido a cada unidad adicional (o valor marginal) de un satisfactor se reduce conforme se consume más de dicho producto; lo anterior debido a que conforme aumenta la cantidad consumida, se baja su utilidad, ya que también se reduce la intensidad de la necesidad. Las consecuencias en el precio que se está dispuesto a pagar por un bien o un servicio se exponen a continuación.

Figura 6. Relación entre precio y utilidad.

$$
\begin{gathered}
Y=f(X) \\
\text { Precio }=\mathrm{f}(\text { Utilidad })
\end{gathered}
$$

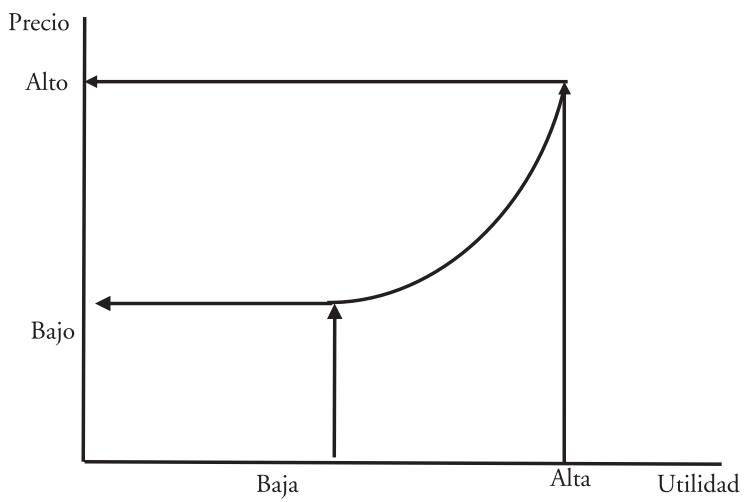

Fuente: elaboración propia.

Una alta utilidad da lugar a la disponibilidad a pagar un mayor precio, en tanto que un producto cuya utilidad sea baja ameritará un precio bajo. Incluso si la utilidad es nula, el consumidor no estará dispuesto a pagar unidad monetaria alguna por él... salvo que 
sea obligado a pagar como sería una multa. Lo descrito se refleja en la relación entre precio y cantidad consumida.

Figura 7. Relación entre precio y cantidad consumida.

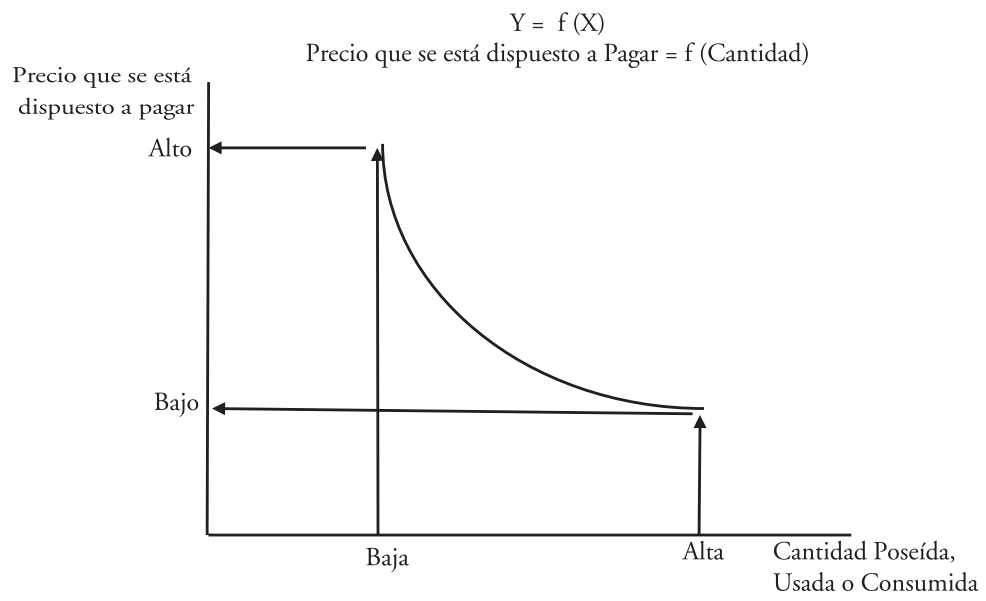

Fuente: elaboración propia.

Para el caso de la gráfica anterior, a mayor cantidad consumida menor el precio reconocido a cada unidad adicional del satisfactor. Cuando la cantidad poseída es baja o nula, la intensidad de la necesidad es alta y por consecuencia la utilidad de una nueva unidad también lo es, lo cual determina un alto valor, lo que se refleja en el precio alto a pagar.

La típica curva de demanda tiene como base al anterior razonamiento:

Figura 8. La cantidad demandada como función inversa al precio.

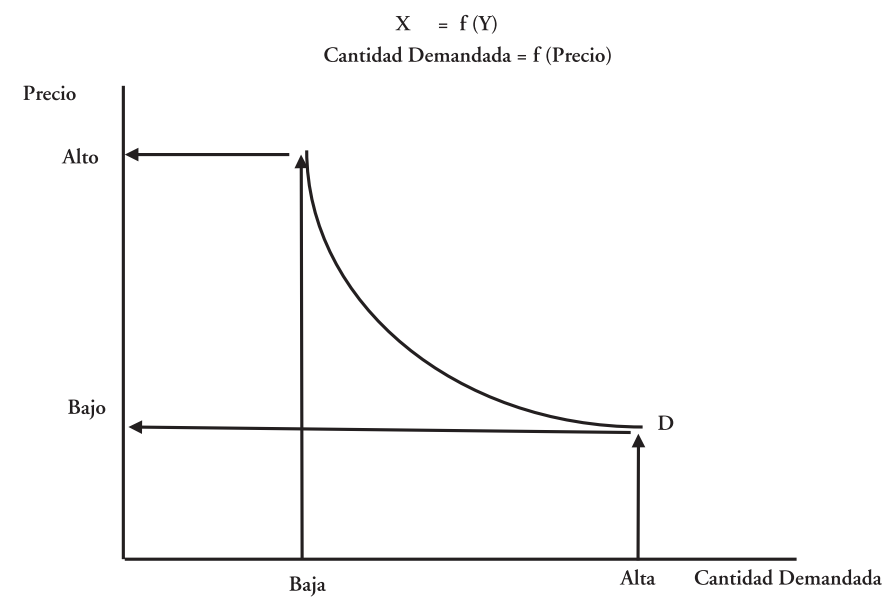

Fuente: elaboración propia. 
El valor percibido por el cliente y el comportamiento del consumidor como constructos paralelos a las Leyes de Gossen / D. Gallegos

En la gráfica previa se describe la "Ley de la demanda. Siempre y cuando las condiciones objetivas y subjetivas no varíen, la cantidad que se demanda de una mercancía varía en proporción inversa al precio; si el precio aumenta, la cantidad demandada disminuye, y si el precio desciende, la cantidad demandada aumenta" (Méndez, 2007:241). Pero como se ha visto, esa curva conlleva una gran cantidad de antecedentes en la intensidad de la necesidad, la utilidad y la valoración.

\section{ii. Segunda Ley de Gossen}

La segunda Ley de Gossen expone la forma en que el consumidor distribuye su gasto de tal manera de sacar el mejor provecho del dinero disponible, ya que "todo individuo que desea satisfacer muchas necesidades de naturaleza diferente con una cantidad fija de gastos debe distribuir estos de forma que el placer producido por cada uno de los diferentes gastos sea el mismo" (James, 1966:187).

Por otra parte, el mejor uso de su recurso lo encuentra cuando la utilidad marginal es proporcional al precio en esa disyuntiva, mediando desde luego el valor que asigna cada persona a cada bien. "... [la] cantidad dada de un bien debe distribuirse entre sus diferentes usos de manera que las utilidades marginales sean iguales en todos sus usos" (James, 1966:187).

Los dos razonamientos anteriores contribuyen a entender la variedad y cantidad de productos que cada consumidor suele decidir adquirir y que está en relación directa de su utilidad y del precio de mercado.

Las leyes de Gossen como explicatorias clásicas de la conducta del consumidor, proponen las maneras básicas en que el consumidor asume la compra de bienes y servicios. Las decisiones de adquirir determinada cantidad y determinada variedad de productos están explicadas por este conjunto de principios que relacionan tanto la intensidad de la necesidad como el precio del producto.

\section{Comportamiento del consumidor mediante ejemplos}

Lo propuesto en el tema anterior, que ha sido la explicación que Gossen da al comportamiento del consumidor (conducta del consumidor en su modelo original), mediante las denominadas Leyes de Gossen, dan pie a la búsqueda de evidencia en hechos actuales de la vida económica cotidiana, muchos de ellos difundidos en los medios.

Las actitudes de aceptación o rechazo de un producto son finalmente el aspecto más importante para todo empresario dado que de ello dependen tanto la realización de la mercancía como los ingresos de la empresa y su posición financiera. "Los mercadólogos 
deben entender cómo es que los consumidores transforman la mercadotecnia y otra información en sus respuestas para comprar" (Kotler y Armstrong, 1996:179).

En todos los procesos de decisión de compra de un producto son determinantes varios aspectos que el oferente deberá tomar en consideración para considerar que está poniendo al alcance del consumidor valor trascendente para él, bajo el supuesto de que “...intervienen cuatro factores psicológicos en la conducta del consumidor. Los factores considerados fueron: la personalidad y el autoconcepto, la motivación, la percepción y el aprendizaje” (Sahui, 2008:1). Entonces, y en base a lo anterior, “...dependiendo de la teoría psicológica que sirva como marco conceptual de los factores antes mencionados, se generarán las estrategias de mercadotecnia que servirán para influir en la conducta de los consumidores" (Sahui, 2008:1).

\section{a) La diferenciación del producto}

El lograr ser diferente a los demás, ya sea por la naturaleza del producto o por los servicios que a él se acompañen, es crucial en la creación de valor para el cliente, siendo tal que hasta "la idea central de los libros que vengo de enumerar -y de este- es poderosamente simple (y también sencillamente poderosa): las cosas "pequeñas" pueden engendrar diferencias enormes, -asombrosamente- grandes en situaciones de vital importancia” (Peters, 2010:7).

La capacidad de complementación de un producto es esencial ya que con ello se logra aumentar su valor y estar así dentro de las preferencias de los consumidores: "parte del declive de BlackBerry se ha producido porque su sistema operativo no ha entrado en el juego de las aplicaciones" (MD Marketing directo, 2011).

Sorprender favorablemente al cliente activo o potencial permite incrementar el valor del producto aunque sea ante algún subsegmento de la población. "Cambiar los colores de un producto sin alterar su esencia. Recuerdo cuando los "Pumas" de la UNAM lanzaron una playera color rosa, con todos los elementos y escudo del equipo, en tiempos en que solíamos asociar a los equipos por sus códigos de colores, lo cual en su momento resultó disruptivo" (Geifman, 2014).

\section{b) La intensificación de necesidades}

La modificación en la intensidad de las necesidades lleva a que un producto tome mayor valor y, por consecuencia, sea más demandado: “... con el descenso de las temperaturas, las personas comienzan a utilizar mucho más los combustibles, especialmente el GLP, cuya demanda actual es de 80.000 kilos, que es equivalente a 8.000 garrafas por día" (La Patria Bolivia, 2012). En ocasiones, incluso, al percibirse como más escaso se acepta un mayor pago por él. 
El valor percibido por el cliente y el comportamiento del consumidor como constructos paralelos a las Leyes de Gossen / D. Gallegos

\section{c) La relación precio-valor}

La percepción de algo es posible a través de los sentidos y conforme mayor cantidad de ellos interviene en el acercamiento a un bien o servicio o incluso en su disfrute, el proceso será mucho más completo. Es por ello que la demostración adquiere una importancia mayúscula para la decisión de adquirir un producto, por lo que "vender por demostración es la mejor forma de acelerar la aceptación de una idea novedosa (= asustadora)" (Peters, 2010:255).

Una relación entre precio y durabilidad de un bien es también una relación entre precio y valor, misma que el cliente percibe como beneficio, así "las ventas de paneles fotovoltaicos y luminarias solares en México se han duplicado en los primeros cinco meses del ańo, debido a una mayor demanda como equipamiento urbano, pues representan un ahorro en costos de luz a mediano plazo, informó la firma mexicana Conermex" (Excélsior, 2013).

\section{d) Los servicios pre y post venta}

Algunos productos se pueden consumir solos o bien junto con otros, siendo entonces complementarios de otros. Al dejarse de consumir aquel producto del cual son complementarios, deja entonces de ser necesario, reduciéndose entonces su utilidad. "Madrid. La crisis económica no perdona a nadie, ni siquiera a la reina mundial de las bebidas refrescantes. Las ventas de Coca Cola en los bares y restaurantes españoles caen un 10\% este año, según reconoce la propia compañía. Este descenso está ocasionado, además de por la menor actividad en los establecimientos de restauración, por la fuerte caída del consumo de cubatas" (Llorente, 2009).

El valor de un producto se ve incrementado por algunos elementos que el consumidor pueda captar como beneficios, lo cual da lugar a una decisión de compra al modificar o acentuar sus preferencias. "Desde el ańo pasado, las tiendas por departamentos han ganado mercado frente a los autoservicios en la venta de ropa y bienes duraderos ofreciendo promociones, crédito sin intereses, más variedad y servicios, según algunos analistas" (El Financiero, 2014).

Las evidencias, a manera de ejemplos, de la vigencia de las Leyes de Gossen son cotidianas y, por ende, innumerables. Es por lo anterior que se hace necesario recuperar su esencia como punto de revisión permanente de aquellas nuevas propuestas que emergen y que pudieran estar tanto en la línea de estas leyes o bien dentro de la discrepancia, y ser aportadoras de nuevas propuestas que contribuyan al estudio del comportamiento del consumidor. 


\section{Conclusiones}

El concepto de conducta del consumidor -tema explorado por la ciencia económicaes factor para entender el comportamiento del consumidor -concepto básico de la mercadotecnia.

Las Leyes de Gossen son explicativas clásicas de la formación del valor y del precio de los bienes y servicios y, por consecuencia, de las decisiones de compra.

Las Leyes de Gossen son referente necesario para comprender el concepto del marketing, de comportamiento del consumidor y de valor percibido por el cliente.

El cabal conocimiento de las Leyes de Gossen permitirá, al estudioso de los procesos mercadológicos, una comprensión más integral de los fenómenos en que se especializa.

El conocimiento del comportamiento del consumidor, como concepto del marketing, amplía sus fronteras al analizarse desde la perspectiva de las Leyes de Gossen.

Finalmente, es importante valorar que las diversas áreas de la mercadotecnia (investigación de mercados, publicidad, promoción, pricing, mercadeo, etc.) podrían tener un mayor desarrollo rescatando las ideas de los cásicos de la economía.

\section{Bibliografía}

Arce, B.; Gallegos, D. y Avendaño, L. (2015). Economía básica. Madrid: Bubock.

Atoche, C. (2010). La conducta del consumidor. En: http://www.cesar-augusto-atoche.com.

Baby, J. y Uribe, J. (2014). "Análisis competitivo por parte de los concesionarios de vehículos automotores en la ciudad de Medellín, mediante el uso del Valor Percibido por el Cliente", Oikos, No. 35, 23-50, Escuela de Administración y Economía, Universidad Católica Silva Enríquez (UCSH), Santiago de Chile.

Carver, Ch. y Sheier, M. (2014). Teorías de la personalidad. Séptima edición. México: Pearson.

El Financiero (2014). Las tiendas departamentales ganan mercado. Fecha consulta: 12/02/2014. En: http://www.elfinanciero.com.mx/.

Excélisor (2013). Aumentan las ventas de panelesfotovoltaicos. Fecha consulta: 23/03/2013. En: http://www.dineroenimagen.com/.

Geifman, Abraham. (2014). La mercadotecnia disruptiva. Mercadotecnia disruptiva. Fecha consulta: 07/02/2014. En: http://www.merca20.com/.

Gómez, C. (s. f.). La revolución marginalista: pioneros y precursores. Apuntes. Alcalá de Henares, Universidad de Alcalá, Departamento de Fundamentos de Economía e Historia Económica. 
El valor percibido por el cliente y el comportamiento del consumidor como constructos paralelos a las Leyes de Gossen / D. Gallegos

Hellriegel, D.; Jackson, S. y Slocum, J. (2004). Administración. Un enfoque basado en competencias. Novena edición, México, Thompson.

Hilarión, J. (2013). Emprendimiento e innovación. Diseña y planea tu negocio. Querétaro, Cengage Learning.

James, E. (1996). Historia del pensamiento económico. México, Fondo de Cultura Económica.

Kotler, P. y Armstrong, G. (1996). Mercadotecnia. Tercera edición. México: Prentice Hall.

La Patria (2012). Aumenta el consumo de gas LP. Bolivia. Fecha consulta: 15/05/2012. En: http://lapatriaenlinea.com/.

Llorente, C. (2009). Las ventas de Coca Cola caen 10\% por el desplome en el consumo de cubatas. El mundo.es. Fecha consulta: 08/06/2009. En: http://www.elmundo.es/mundodinero/

Martín, D. Barroso, C. y Martín, E. (s. f.). El valor percibido de un servicio. Revista española de investigación de Marketing ESIC. Sevilla: Universidad de Sevilla.

MD Marketing directo (2011). BlackBerry cae en desuso por no entrar en la batalla de las aplicaciones. Fecha consulta: 09/06/2011. En: http://www.marketingdirecto.com/.

Méndez, J. (2007). La economía en la empresa. Tercera edición, México, McGrawHill. Parkin, M. (2014). Economía. Décima primera edición, México, Parkin.

Peters, T. (2010). Las pequeñas grandes cosas. 163 maneras para alcanzar la excelencia. Bogotá, Grupo Editorial Norma.

Ramos, F. (2004). El comportamiento del consumidor a través del concepto demanda. Universidad de Puerto Rico en Huamacao: Centro de Competencia de la Comunicación. Fecha consulta: 23/08/2013. En: http://www1.uprh.edu.

Robles, G. y Alcérreca, C. (2000). Administración. Un enfoque interdisciplinario. México, Pearson-Prentice Hall.

Sahui, J. (2008). Influencia de los factores psicológicos en la conducta del consumidor. Tecsistecatl. Revista electrónica de Ciencias Sociales. Número 5, diciembre 2008. Fecha consulta: 07/02/2014. En: http:/www.eumed.net/rev/tecsistecatl/n 5 .

Schifman, L. y Kanuk, L. (2010). Comportamiento del consumidor. Octava edición, México, Pearson-Prentice Hall.

Siliceo, V. (s. f.). Comportamiento del consumidor. Fecha consulta: 18/03/2014. En: http://brd.unid.edu.mx/recursos. 
Stanton, W.; Etzel, M. y Walker, B. (2000). Fundamentos de marketing. Undécima edición, México, McGraw-Hill.

\section{Cómo citar este artículo:}

Gallegos, Divvio (2015). "El valor percibido por el cliente y el comportamiento del consumidor como constructos paralelos a las Leyes de Gossen", Oikos No 41, 89-107, Escuela de Administración y Economía, Universidad Católica Silva Henríquez (UCSH), Santiago de Chile.

[http://ediciones.ucsh.cl/revistas.php]

Fecha de recepción: 31/08/2015

Fecha de aceptación: 19/02/2016 